\title{
Cerebral Pachyleptomeningitis Associated with MPO-ANCA Induced by PTU Therapy
}

\author{
Takato Abe ${ }^{1}$, Shigeru Nogawa ${ }^{1}$, Norio Tanahashi ${ }^{1}$, \\ Junichi Shiraishi $^{2}$, Eiji Ikeda ${ }^{2}$ and Norihiro Suzuki ${ }^{1}$
}

\begin{abstract}
A 34-year-old woman with hyperthyroidism, who had been previously treated with propylthiouracil (PTU) is reported. She was admitted because of clumsiness in her left hand and abnormal behavior. A neurological examination demonstrated impairment of higher cortical function, and weakness and hyperreflexia of the left leg. An MRI scan with gadolinium enhancement showed pachyleptomeningeal thickening in the right frontoparietal lobe. Blood tests revealed a high MPO-ANCA titer of $122 \mathrm{EU}$ (normal:<10 EU). After steroid administration, the neurological symptoms and the MRI findings improved markedly. This is the first report of PTU-induced cerebral pachyleptomeningitis associated with a high serum MPO-ANCA titer.
\end{abstract}

Key words: PTU, MPO-ANCA, pachyleptomeningitis

(DOI: 10.2169/internalmedicine.46.1859)

\section{Introduction}

Hypertrophic pachymeningitis is a rare condition characterized by thickening of the pachymeninges. Recently, some cases of hypertrophic pachymeningitis have been described as being associated with a high myeloperoxidase antineutrophil cytoplasmic antibody (MPO-ANCA) titer. Although MPO-ANCA positive pachymeningitis has been reported to occur in patients who have a variety of underlying diseases, such as Wegener granulomatosis $(1,2)$, Sjögren syndrome (3), rheumatoid arthritis (4), and microscopic polyangitis $(5,6)$; most cases are reported as idiopathic (7). On the other hand, the antithyroid drug propylthiouracil (PTU) has been reported to be capable of triggering MPOANCA elevation, and MPO-ANCA-associated vasculitis sometimes occurs in patients treated with PTU $(8,9)$.

We report a case of cerebral pachyleptomeningitis associated with a high serum MPO-ANCA titer, induced by PTU. Both the discontinuation of PTU therapy and the administration of steroid therapy were thought to have been responsible for the resolution of the higher cortical symptoms and reversal of the meningeal thickening, presumably by reducing the serum MPO-ANCA titer.

\section{Case Report}

In January 2003, a 34-year-old woman who had been treated with PTU for Basedow's disease since 23 years of age lost consciousness and fell from her bicycle. Within the next 2 weeks, she noticed clumsiness of her left hand, and she also exhibited abnormal behavior, such as burning cloth on a stove and forgetting how to flush the toilet. She did not complain of a headache or fever. She consulted a psychologist and was diagnosed with depression. An antidepressant drug was prescribed, but was ineffective. The dose of PTU had varied between 0 and $400 \mathrm{mg}$ daily over a period of 11 years, but it was replaced with methimazole $20 \mathrm{mg}$ daily 20 days after she lost consciousness, because her hyperthyroidism had worsened.

In February, the patient was admitted to our hospital for further evaluation and treatment. Physical examination on admission revealed exophthalmos and a mild goiter. She seemed to be alert, but had visual hallucinations and delusions. Examination of higher cortical activity revealed dressing apraxia, topographical agnosia, prosopagnosia, and slight cognitive impairment. Neurological examination revealed graphanesthesia, astereognosis, weakness of the left arm, and hyperreflexia of the left leg. Bilateral incomplete exter-

\footnotetext{
${ }^{1}$ Neurology, Keio University School of Medicine, Tokyo and ${ }^{2}$ Pathology, Keio University School of Medicine, Tokyo Received for publication April 11, 2006; Accepted for publication October 13, 2006 Correspondence to Dr. Takato Abe, snogawa@tdc.ac.jp
} 


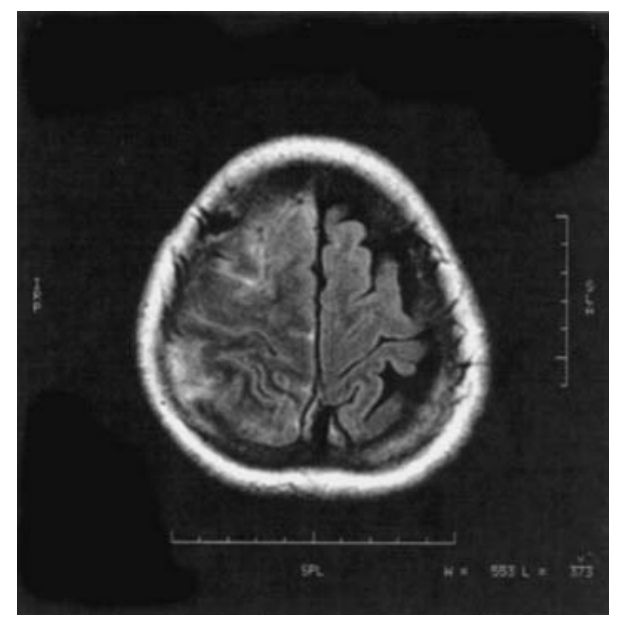

Figure 1. A high intensity signal was seen on the surface of the right frontal and parietal cortex in the axial-view MRI scan (FLAIR image) acquired five days after admission. The left cerebral hemisphere seems to be atrophic compared to the right side.

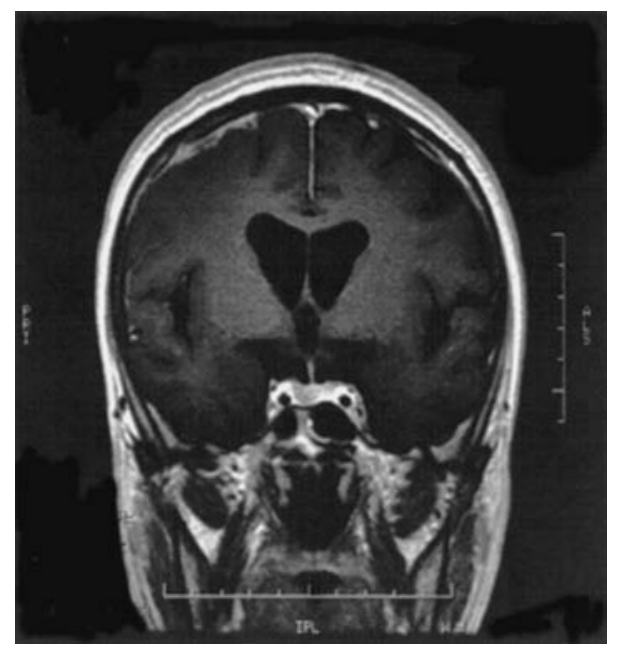

Figure 2. The coronal view (T1 image after gadolinium injection) revealed left cerebral hemisphere atrophy and thickening with enhancement of both the dura and pia mater, predominantly on the right side.

nal ophthalmoplegia with limitation of eye movements in all directions secondary to Graves' ophthalmopathy was also diagnosed. No sensory impairment was revealed. Blood tests showed no abnormality in the peripheral blood count (WBC

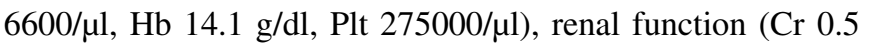
$\mathrm{mg} / \mathrm{dl})$ and CRP (0.12 mg/dl). TSH level was decreased to $0.01 \mathrm{mU} / \mathrm{ml}$ and MPO-ANCA titer was elevated to $122 \mathrm{EU}$ (normal: $<10 \mathrm{EU}$ ). The cytoplasmic anti-neutrophil cytoplasmic antibody (C-ANCA) titer was less than $10 \mathrm{U} / \mathrm{ml}$. Cerebrospinal fluid (CSF) analysis revealed a cell count of $4 / \mathrm{mm}^{3}$ (all monocytes), an elevated protein concentration of $52 \mathrm{mg} / \mathrm{dl}$, glucose level of $53 \mathrm{mg} / \mathrm{dl}(58 \%$ of the simultaneous serum value), and an elevated $\mathrm{IgG}$ level of $16.8 \mathrm{mg}$ / dl. CSF cultures for bacteria, fungi, and acid-fast bacilli showed no growth. The CSF IgG index was elevated to

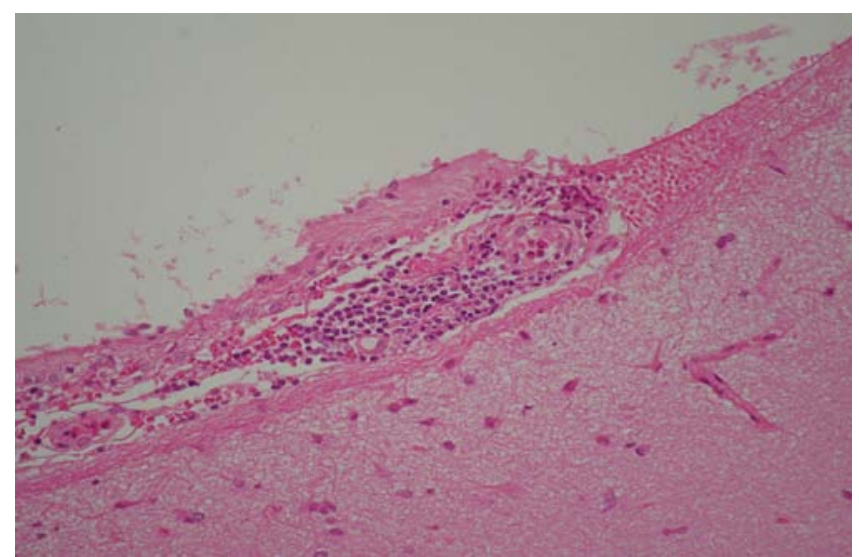

Figure 3. Inflammatory cell infiltration, comprised of lymphocytes and a small number of neutrophils, was seen around the small vessels in the subarachnoid space. However, there was no obvious finding of vasculitis. (HE stain, $\times 200$ )

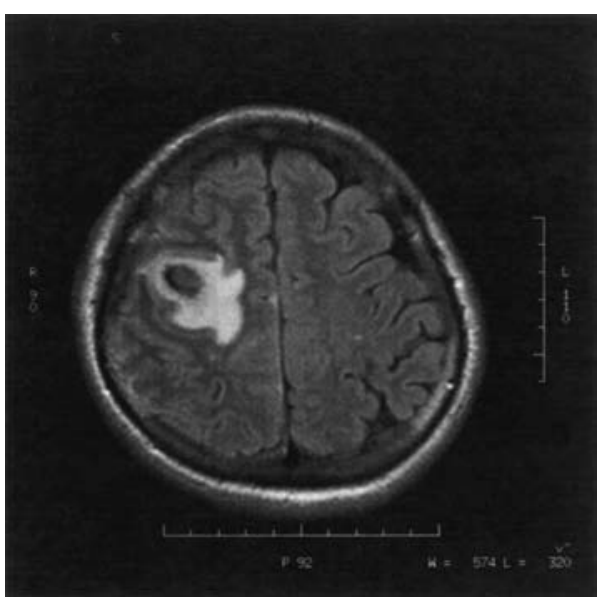

Figure 4. The MRI image of three days after biopsy (FLAIR image) revealed a high intensity lesion with low intensity inside in the right front-parietal lobe, suggesting venous infarction.

1.48. Chest and abdominal CT did not disclose any abnormal findings. An MRI scan showed pachyleptomeningeal thickening with gadolinium enhancement in the right frontoparietal area with moderate atrophy of left temporal regions (Figs. 1, 2). A needle biopsy of the dura mater and cortex was performed in the right frontoparietal area, but no significant pathological changes were detected in the brain parenchyma or dura mater. Inflammatory cell infiltration comprising lymphocytes and a small number of neutrophils was noted around the small vessels in the subarachnoid space, but no clear evidence of vasculitis was observed (Fig. 3).

Three days after the biopsy, the weakness in the left arm had become more severe, and brain CT and MRI scans revealed venous infarction around the biopsy site (Fig. 4). We treated the patient with dexamethasone $8 \mathrm{mg}$ daily i.v. for four days, and then with prednisolone $30 \mathrm{mg}$ p.o. daily. A relatively smaller dose of PSL than usual was administrated, 


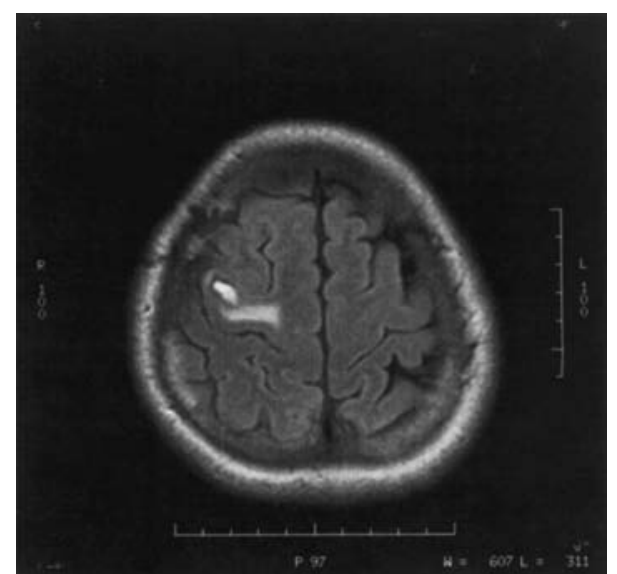

Figure 5. In the MRI image at almost one month after biopsy (FLAIR image), venous infarction and high intensity of brain hemisphere was improved.

because we could not obtain sufficient evidence of vasculitis from the pathological specimen. However, the neurological symptoms and the MRI findings improved markedly in response to treatment (Fig. 5), and two months after admission the patient was discharged almost symptom-free. Three months later the MPO-ANCA titer had fallen to 54 EU.

\section{Discussion}

Since Stankus and Johnson (10) first described ANCApositive vasculitis in a patient receiving antithyroid medication in 1992, about 100 cases of MPO-ANCA elevation induced by PTU have been reported, and most of them have been in Japan. PTU has been shown to accumulate in polymorphonuclear leukocytes (11) and alter the structure of myeloperoxidase (12), and that has been speculated to be the mechanism of the abnormal production of MPO-ANCA.

This is the first report of a case of cerebral pachylep- tomeningitis induced by PTU in which the symptoms and meningeal thickening were demonstrated to be closely associated with the serum MPO-ANCA titer. Although the images of this case were different from these of typical pachymeningitis since our case also showed robust leptomeningeal thickening, some similar etiologic mechanism by MPOANCA might also underlie the occurrence of pachyleptomeningitis. There have been only two previous reports of MPO-ANCA-positive pachymeningitis occurring in a patient receiving PTU therapy. Ito et al (13) reported a case of MPO-ANCA-positive spinal pachymeningitis in a patient who had taken PTU for 4 years for Graves' disease, and histological examination of the spinal meninges revealed non-specific chronic inflammation. Takahashi et al (14) reported a case of Graves' disease that became complicated by cranial pachymeningitis after PTU treatment, but they did not mention any role of PTU in their case, and they did not state whether PTU was discontinued (15). Thus, the relationships among PTU, MPO-ANCA, and pachymeningitis remain unclear in this report.

Thickening of both the dura and pia mater was observed on the brain MRI images in the present case. Although no definite evidence of inflammation was detected in the small needlebiopsy specimen, leukocyte infiltration into the subarachnoid space was confirmed, corresponding to the pachyleptomeningeal thickening observed by MRI. Brain atrophy might be associated with long lasting thyroid dysfunction and the laterality might be due to the edema around pachyleptomeningitis.

Whenever a patient taking PTU develops neurological deficits, the serum MPO-ANCA titer should be measured and brain MRI should be performed to exclude pachyleptomeningitis. Administration of steroid and discontinuation of PTU might be effective for patients of MPO-ANCApositive pachyleptomeningitis induced by PTU.

\section{References}

1. Case records of the Massachusetts General Hospital. Weekly clinicopathological exercises. Case 9-1999. A 74-year-old woman with hydrocephalus and pleocytosis. N Engl J Med 340: 945-953, 1999.

2. Nagashima T, Maguchi S, Terayama $\mathrm{Y}$, et al. P-ANCA-positive Wegener's granulomatosis presenting with hypertrophic pachymeningitis and multiple cranial neuropathies : case report and review of literature. Neuropathology 20: 23-30, 2000.

3. Yoritaka A, Tsukamoto T, Ohta K, Kishida S. A clinical study of pachymeningitis. No To Shinkei (Brain and Nerve) 54: 235-240, 2002 (in Japanese, Abstract in English).

4. Funauchi M, Yoo BS, Sugiyama M, et al. A case of rheumatoid meningitis positive for perinuclear antineutrophil cytoplasmic antibody. Ann Rheum Dis 59: 1001-1002, 2000.

5. Kono H, Inokuma S, Nakayama H, Yamazaki J. Pachymeningitis in microscopic polyangiitis (MPA) : a case report and a review of central nervous system involvement in MPA. Clin Exp Rheumatol 18: 397-400, 2000.

6. Furukawa Y, Matsumoto Y, Yamada M. Hypertrophic pachymeningitis as an initial and cardinal manifestation of microscopic polyangiitis. Neurology 63: 1722-1724, 2004.
7. Yakushiji Y, Kurohara K, Toda S, Abe M, Kuroda Y. A case of hypertrophic spinal pachymeningitis associated with MPO-ANCA. Rinsho Shinkeigaku 42: 873-877, 2002 (in Japanese, Abstract in English).

8. Kitahara T, Hiromura K, Maezawa A, et al. Case of propylthiouracil-induced vasculitis associated with anti-neutrophil cytoplasmic antibody (ANCA) ; review of literature. Clin Nephrol 47: 336-340, 1997.

9. Dolman KM, Gans RO, Vervaat TJ, et al. Vasculitis and antineutrophil cytoplasmic autoantibodies associated with propylthiouracil therapy. Lancet 342: 651-652, 1993.

10. Stankus SJ, Johnson NT. Propylthiouracil-induced hypersensitivity vasculitis presenting as respiratory failure. Chest 102: 1595-1596, 1992.

11. Lam DC, Lindsay RH. Accumulation of 2-[ $\left.{ }^{[4} \mathrm{C}\right]$ propylthiouracil in human polymorphonuclear leukocytes. Biochem Pharmacol 28: 2289-2296, 1979.

12. Lee E, Hirouchi M, Hosokawa M, Sayo H, Kohno M, Kariya K. Inactivation of peroxidases of rat bone marrow by repeated administration of propylthiouracil is accompanied by a change in the heme structure. Biochem Pharmacol 37: 2151-2153, 1988. 
DOI: $10.2169 /$ internalmedicine.46.1859

13. Ito K, Fujii H, Sumitomo H, et al. A case of spinal pachymeningitis associated with MPO-ANCA during treatment with antithyroid drug (abstract). Nihon Jinzo Gakkaishi 36: 1354, 1994 (in Japanese).

14. Takahashi K, Kobayashi S, Okada K, Yamaguchi S. Pachymeningitis with a perinuclear antineutrophil cytoplasmic antibody : re- sponse to pulse steroid. Neurology 50: 1190-1191, 1998.

15. Meilof JF. p-ANCA and hypertrophic pachymeningitis. Neurology 52: 430-431, 1999.

(C) 2007 The Japanese Society of Internal Medicine http://www.naika.or.jp/imindex.html 